Saudi Journal of Oral and Dental Research

Abbreviated Key Title: Saudi J Oral Dent Res

ISSN 2518-1300 (Print) |ISSN 2518-1297 (Online)

Scholars Middle East Publishers, Dubai, United Arab Emirates

Journal homepage: https://saudijournals.com/sjodr

Original Research Article

\title{
Knowledge of CAD/ CAM or Conventional Ceramic Material Restorations in Prosthodontics: A Qualitative Research
}

Dr. Karim Aboukoura ${ }^{1 *}$, Dr. Sangeetha Karunakaran ${ }^{2}$, Dr. Malti Tulli ${ }^{3}$, Dr. Turfa Zubedi ${ }^{4}$, Dr. Chaitaliben Gandhi ${ }^{5}$, Dr. Nuba Afzal Siddiqi ${ }^{6}$

${ }^{1}$ BDS, Orlando, United States

${ }^{2}$ BDS, Sri Ramakrishna Dental College and Hospital, Coimbatore, Tamil Nadu, India

${ }^{3}$ BDS, Guru Nanak Dev Dental College and Research Institutes, Sunam, Punjab, India

${ }^{4}$ B.D.S, Meenakshi Ammal Dental College, Chennai, Tamil Nadu, India

${ }^{5}$ BDS, Government Dental College, Civil Hospital Campus, Civil Hospital Rd, Asarwa, Ahmedabad, Gujarat, India

${ }^{6}$ BDS, Uttar Pradesh Dental College, Lucknow, India

DOI: $10.36348 /$ sjodr.2020.v05i04.003

| Received: 01.04.2020 | Accepted: 13.04.2020 | Published: 15.04.2020

*Corresponding author: Dr. Karim Aboukoura

Abstract

Aim of study: The purpose of the study is to understand the knowledge of CAD/CAM amongst various prosthodontists and the materials that they use while fabricating restorations considering the features of this technology. Methodology of study: A questionnaire survey was conducted over an year amongst 40 prosthodontists about the CAD/CAM technology, the materials they generally use to fabricate the restorations and how often they use this technology in their daily practice. Results: Mostly feldspathic porcelain was used in inlays and onlays as well as veneers (76\%) whereas only a fraction of prosthodontists used lithium disilicate based materials. In anterior three-unit restorations, many options were considered from porcelain fused to metal $(54 \%)$, lithium disilicate based materials (28\%), to others using cubic zirconia also. In multi- unit posterior restorations, full metal was the commonest (63\%) as compared to full contour zirconia. Conclusion: With the advancement taking place digital systems and CAD/ CAM has the potential to change the conventional technologies.

Keywords CAD/CAM, Ceramics, esthetic restoration.

Copyright @ 2020: This is an open-access article distributed under the terms of the Creative Commons Attribution license which permits unrestricted use, distribution, and reproduction in any medium for non-commercial use (NonCommercial, or CC-BY-NC) provided the original author and source are credited.

\section{INTRODUCTION}

Over the past decades, main technological advances in dentistry have fetched practitioners improved equipment, techniques, and materials. One of those add-ons has been chairside computer-aided design and computer-aided manufacturing (CAD-CAM) [1]. Dental CAD/CAM technology is gaining momentum because of its advantages in terms of time consuming, materials savings, standardisation of the fabrication process, and probability of the restorations. The number of steps essential for the fabrication of a restoration is a smaller amount as compared to traditional methods. Additional benefit of CAD/CAM dentistry includes the use of new materials and data acquisition, which signifies a non-destructive method of saving impressions, restorations and information that is saved in a computer and establishes an extraordinary communication tool for assessment. The integration of dental technology has not only brought a new range of manufacturing methods and material options, but also some apprehensions about the processes involving restorations fit, quality, precision, short and long-term prognosis [2]. Skills vary with each practitioner as a outcome of individual practice styles, team members and patient outlooks, CAD/CAM technology may not be right for everyone. Practitioners must reflect a variety of factors when making a CAD/CAM purchase: their own knowledge of and accomplishment with bonded restorations and their preparation design, the need for patient education, team members reception of the system, negative laboratory experiences, their capability to accept the defined treatment protocol, their ability to accept the application of computer technology and the integration of the system into the office and the daily practice [3].

Practitioners can select from feldspathic porcelain, leucite reinforced porcelain and resin. These materials come in a variety of shades, colour gradients and translucencies, which permits for better aesthetic matching to the patient's dentition. Practitioners can attain even greater detail and occlusal characteristics over staining application to the superficial ceramic layer 
that then can be glazed and fired. Manufacturers are fabricating all-ceramic and resin-based composite blocks that offer a dense restorative product without inadequacies. The use of these porcelain and resinbased blocks delivers quality control, reduces polymerization shrinkage and confines this measurable property to the resin cement that is used [3]. The overall method to CAD/CAM technology and the choices that border each restoration have become easier and more foreseeable through improved milling software, restoration design options and material selection. In particular, the software has become much simpler and the initial learning curve has improved significantly in the last five years. CEREC's (computer assisted ceramic reconstruction) software suggestions the capability to design a restoration, copy an existing tooth and regulate the occlusion virtually. Most restoration designs can be accomplished in less than five minutes and then milled in 10 to 12 minutes (Figure-1). Recent software enhancements have reduced the milling time by about 30 percent. As clinicians gain confidence and become more capable in producing single posterior restorations, they will be able to offer gradually more advanced or aesthetically challenging restorations for their patients who need them. In core, the clinician syndicates computer-aided technology with artistry [3]. CAD/CAM technology was formed to overcome several disadvantages associated with the conventional lost wax techniques. The first was to remove shrinkage and expansions associated with wax and models. The second was to remove shrinkage and porosities related to casting procedure. The third was to make restoration of tooth easier, quicker, and more accurate in terms of precision and fit. In most cases CAD/CAM technology can used to offer restorations to patients within the same day. More than 30,000 dentists around the world own scanning and milling machines [4].

Fig-1: CAD/CAM technology workflow to fabricate restorations

\begin{tabular}{l}
$\begin{array}{l}\text { Recording intra-oral conditions to a } \\
\text { computer software program using intra- } \\
\text { oral scanner or camera. }\end{array}$ \\
$\begin{array}{l}\text { Manipulating digital data in the design } \\
\text { sequence using software program to } \\
\text { create the desired contours, occlusion } \\
\text { and contacts of the restoration. }\end{array}$ \\
$\begin{array}{l}\text { Fabricating the desired restoration using } \\
\text { subtractive manufacturing technology } \\
\text { process to shape or mill the desired } \\
\text { restoration contour from a preformed } \\
\text { block of material based on volumetric } \\
\text { design created with software program. }\end{array}$ \\
\hline
\end{tabular}

\section{AIM OF THE STUDY}

The purpose of the study is to understand the knowledge of CAD/CAM amongst various prosthodontists and the materials that they use while fabricating restorations considering the features of this technology.

\section{METHODOLOGY OF STUDY}

A questionnaire survey was conducted over an year amongst 40 prosthodontists about the CAD/CAM technology, the materials they generally use to fabricate the restorations and how often they use this technology in their daily practice. An email was sent to them with an open-ended format in English language and their replies were recorded on a spreadsheet and it was analyzed using descriptive statistics.

\section{RESULTS}

The results varied in terms of the situations where the restorations were used, based on location, esthetic considerations, strength based on resistance to occlusal forces, stability and resilience. Mostly feldspathic porcelain was used in inlays and onlays as well as veneers $(76 \%)$ whereas only a fraction of prosthodontists used lithium disilicate based materials (Table-1). In cases of single crown restoration with 
porcelain using $\mathrm{CAD} / \mathrm{CAM}$ technology, the prosthodontists preferred porcelain fused to metal or leucite reinforced based materials rather than lithium disilicate which provided more strength. In case of posterior single crown, usage of metal crowns or porcelain fused to metal was used more, only few used full zirconia crowns since the cost of producing it as well technique sensitivity was on the higher side. In anterior three-unit restorations, many options were considered from porcelain fused to metal (54\%), lithium disilicate based materials (28\%), to others using cubic zirconia also. In multi- unit posterior restorations, full metal was the commonest (63\%) as compared to full contour zirconia.

Table-1: Ceramic CAD/CAM materials used by different prosthodontists

\begin{tabular}{|c|c|c|c|c|c|c|c|}
\hline CAD/CAM materials & $\begin{array}{l}\text { Feldspathic/leucite } \\
\text { reinforced porcelain }\end{array}$ & $\begin{array}{l}\text { Lithium } \\
\text { disilicate based } \\
\text { material }\end{array}$ & $\begin{array}{l}\text { Resin based } \\
\text { composite }\end{array}$ & $\begin{array}{l}\text { Cubic } \\
\text { zirconia }\end{array}$ & PFM & $\begin{array}{l}\text { Full } \\
\text { contour } \\
\text { zirconia }\end{array}$ & $\begin{array}{l}\text { Metal } \\
\text { crowns }\end{array}$ \\
\hline Inlay/ Onlay/ Veneer & $76 \%$ & $18 \%$ & $4 \%$ & - & $2 \%$ & - & - \\
\hline Anterior single crown & $30 \%$ & $25 \%$ & - & - & $45 \%$ & - & - \\
\hline Posterior single crown & $8 \%$ & - & - & - & $25 \%$ & $10 \%$ & $57 \%$ \\
\hline Anterior three-unit & $10 \%$ & $28 \%$ & - & $8 \%$ & $54 \%$ & - & - \\
\hline Posterior three unit & $5 \%$ & - & - & - & $12 \%$ & $20 \%$ & $63 \%$ \\
\hline
\end{tabular}

\section{DISCUSSION}

Developments in computer-aided design (CAD) / computer-aided manufacturing (CAM) technologies and their ease of application permitted the development of original treatment concepts for modern prosthodontics. This recent standard shift in fixed prosthodontics from traditional to marginally invasive treatment approaches is demonstrated by the clinical long-term success of bonded CAD/CAM glass-ceramic restorations. Today, defect-oriented restorations, such as inlays, onlays, and posterior crowns, are mostly fabricated from glass-ceramics in monolithic application [5]. The variety of $\mathrm{CAD} / \mathrm{CAM}$ ceramic restorative systems is regularly evolving to meet the bigger demands for highly aesthetic, biocompatible, and long-lasting restorations. Recently presented polymerinfiltrated ceramic network CAD/CAM blocks add innovative treatment options in CAD/CAM chairsidevisit restorations. The material-specific high-edge firmness allows the CAD/CAM machinability of thin restoration margins. Full-contour zirconia restorations are regularly gaining market portion at the expense of bilayered systems. Developments in material science and bonding protocols foster the expansion of novel material combinations or fabrication techniques of established high-strength zirconia ceramics. CAD/CAM applications bid a standardized manufacturing process resulting in a consistent, predictable, and economic workflow for individual and complex teeth-supported restorations [5]. Along with novel materials, there has been a instantaneous advancement in fabrication techniques for ceramic restorations. There has been a change from the traditional hand layering or powdercondensation technique to CAD/CAM technology. With $\mathrm{CAD} / \mathrm{CAM}$ systems, the oral environment is apprehended using intraoral or laboratory scanners, and the data is used to design and mill the restoration digitally. This process decreases human blunder and labour, and can allow for faster, more steady restoration processing. Unlike traditional methods, CAD/CAM materials are accessible as "green stage," or partially sintered blanks, which are porous and enable fast cutting without fracture. Once milled, further sintering is necessary to obtain the material's desired mechanical properties [6]. Full-contour zirconia is a favored choice of many practitioners for molar single crowns and posterior multi-unit bridges. It can offer an alternative to gold crowns and Porcelain Fused to Porcelain (PFM) restorations. In case of Inlays, Onlays and Veneers, most materials meet the minimum strength prerequisite of $50 \mathrm{MPa}$ for Inlays, Onlays and Veneers. Though, adhesive cementation and aesthetics are vital factors, and among those to reflect when selecting a material. Lithium Di-silicate (LDS) and Resin Ceramic Composite (RCC) are frequently chosen for several reasons: LDS provide improved mechanical properties and aesthetics, while RCC is easy to fabricate and requires less chair time [6]. For anterior single crowns, LDS restorations deliver an excellent blend of aesthetics and strength. They offer better mechanical properties than feldspathic or leucite-reinforced porcelains and improved aesthetics associated to the traditional PFM [6]. With its increased strength and less cost, full-contour zirconia provides a substitute to conventional full-metal crowns and PFMs. As a single layer restoration, it needs less manufacturing time than PFM, but delivers less translucency. LDS is favoured for low retention preparations over zirconia because of its improved bonding, and should also be measured for patients whose wide smiles call for a more natural appearance in the molar region [6]. The evolution, features, and marginal fit of milled CAD/CAM allceramic restorations materials and systems display that it is possible to fabricate restorations with the same marginal fit anticipated from conventional methods and within the range of clinically accepted restorations. When linking both methods, the advantage of using CAD/CAM technology is not to obtain the most detailed level of fit, but rather to attain a high level of reliability in a large number of restorations; especially when high production levels are expected [2]. Advantages of a laboratory CAD/CAM milled restoration comprise of reduced chairside time and increased precision. Since a stone model is not used, 
stone pouring blunders are eliminated, as are errors related with abrasion of the adjacent and opposing teeth due to manipulation of the models through fabrication that could result in over-contouring, tight contacts, and excessive occlusal height. In addition, less time is required for fabrication of the substructure. Depending on the laboratory, it may be more profitable to subcontract CAD/CAM milling to a different laboratory. The laboratory would then focus on the challenging artistic process of enhancing the ultimate contour and aesthetics of the restoration [7].

\section{CONCLUSION}

In current scenario CAD/CAM technology has become a core part of dentistry with a greater number of restorations being fabricated. With the advancement taking place digital systems and CAD/ CAM has the potential to change the conventional technologies. With these restorations can be fabricated rapidly, with better properties and aesthetic demands of the patient.

\section{REFERENCES}

1. Blackburn, C., Rask, H., \& Awada, A. (2018). Mechanical properties of resin-ceramic CADCAM materials after accelerated aging. The Journal of prosthetic dentistry, 119(6), 954-958.
2. Brenes, C., Duqum, I., \& Mendonza, G. (2016). Materials and systems for all ceramic CAD/CAM restorations. Dental Tribune. CAD/CAM international magazine of digital dentistry, (3):1015.

https://www.researchgate.net/publication/3162146 17

3. Trost, L., Stines, S., \& Burt, L. (2006). Making informed decisions about incorporating a CAD/CAM system into dental practice. The Journal of the American Dental Association, 137, 32S-36S.

4. Culp, L., \& Touchstone, A. (2006). CAD/CAM Dentistry: A new forum for dentist-technician Teamwork. Inside Dent, 2(7).

5. Spitznagel, F. A., Boldt, J., \& Gierthmuehlen, P. C. (2018). CAD/CAM ceramic restorative materials for natural teeth. Journal of dental research, 97(10), 1082-1091.

6. Luiz, M. (2017). Ceramic CAD/CAM Materials: An Overview of Clinical Uses and Considerations. ADA Professional Product Review, 12(1):1-9.

7. Klim, J., \& Corrales, E. B. (2009). Innovation in Dentistry: CAD/CAM Restorative Procedures. ineedce. Com. 